\title{
Initiation of Pharyngeal Swallow with Bolus Head at Posterior Angle of Ramus
}

National Cancer Institute

\section{Source}

National Cancer Institute. Initiation of Pharyngeal Swallow with Bolus Head at Posterior

Angle of Ramus. NCI Thesaurus. Code C127220.

A finding of pharyngeal swallow initiated with bolus head at posterior angle of ramus. 